\title{
Comparison Between Moiré Sampling Scanning Transmission Electron Microscopy Geometrical Phase Analysis Strain Characterization Method and Dark-Field Electron Holography
}

Alexandre Pofelski ${ }^{1}$, Viraj Whabi ${ }^{2}$, Shahram Ghanad-Tavakoli ${ }^{1}$ and Gianluigi Botton ${ }^{3}$

${ }^{1}$ McMaster University, Canada, ${ }^{2}$ McMaster University, United States, ${ }^{3}$ Department of Materials Science and Engineering, McMaster University, Hamilton, ON, Canada, Canada

Moiré artifacts from undersampling are mostly considered as nuisance in digital imaging. However, recent work demonstrated that the Moiré sampling concept [1,2] can be exploited in Scanning Transmission Electron Microscopy (STEM) to characterize the deformation in a crystalline material $[3,4,5,6]$. By intentionally undersampling the crystalline lattices, the scanning grid of the electron beam raster interferes with the crystal lattices creating a STEM Moiré hologram embedding the deformation field in the Moiré fringes' spatial variations. As the undersampling condition is met for large pixel spacings, the STEM Moiré hologram field-of-view can be extended to a few microns, reaching the capabilities of both the Nano Beam (Precession) Electron Diffraction (NB(P)ED) and the Dark Field Electron Holography (DFEH) strain characterization methods. Very often comparisons between similar, but not identical, methods provide valuable insights. For example, an experimental match between the established HRSTEM Geometric Phase Analysis method (HR-STEM GPA) and the Moiré sampling Scanning Transmission Electron Microscopy Geometrical Phase Analysis technique (STEM Moiré GPA) was recently demonstrated [7] and provides confidence in using the recently developed method. The reliability of STEM Moiré GPA was further confirmed with another experimental match with the NB(P)ED technique [8].

In this study, a comparison between STEM Moiré GPA and DFEH is presented to overcome the field-ofview limitation of the HR-STEM GPA method and to evaluate the experimental results from two different contrast mechanisms (STEM vs Conventional Transmission Electron Microscopy (CTEM)). Fig. 1 presents the strain measurement results obtained on a InP/InAs0.35 $\mathrm{P}_{0.65} / \mathrm{InP}$ stack grown by Molecular Beam Epitaxy. STEM Moiré GPA and DFEH results are, globally, in good agreement with each other but statistically relevant small differences are nonetheless present. The discrepancies suggest that different strain states in the same sample were measured. Since strain relaxation causes the strain distribution to be non-uniform along the beam propagation direction, the disparities between the strain characterization methods can be interpreted as a depth-of-field (DOF) and depth sensitivity difference. Mechanical Finite Element Method (FEM) simulations modelling the strain relaxation from a $105 \mathrm{~nm}$ thick foil consisting of a InP/InAs $0.35 \mathrm{P}_{0.65} / \mathrm{InP}$ stack are presented in Fig. 2. The best consistency between simulated and experimental data is found when STEM Moiré GPA DOF is chosen to be narrow $(1 \mathrm{~nm})$ and located near the top surface of the lamella ( $3 \mathrm{~nm}$ below the surface). For DFEH, a DOF of half of the lamella thickness located in the center of the lamella shows the closest match with the FEM results. The difference in depth locations and DOFs seems to be reasonable when considering the contrast mechanism of STEM and DFEH. STEM is known to have a narrow DOF (a few nanometers depending on the experimental parameters [9]), while CTEM (related to DFEH) accounts for the entire thickness of the lamella with a non-uniform phase propagation [10]. This study therefore emphasizes on the necessity to properly account for the properties of each strain characterization method and the impact of strain relaxation on the experimental results $[11,12]$. 

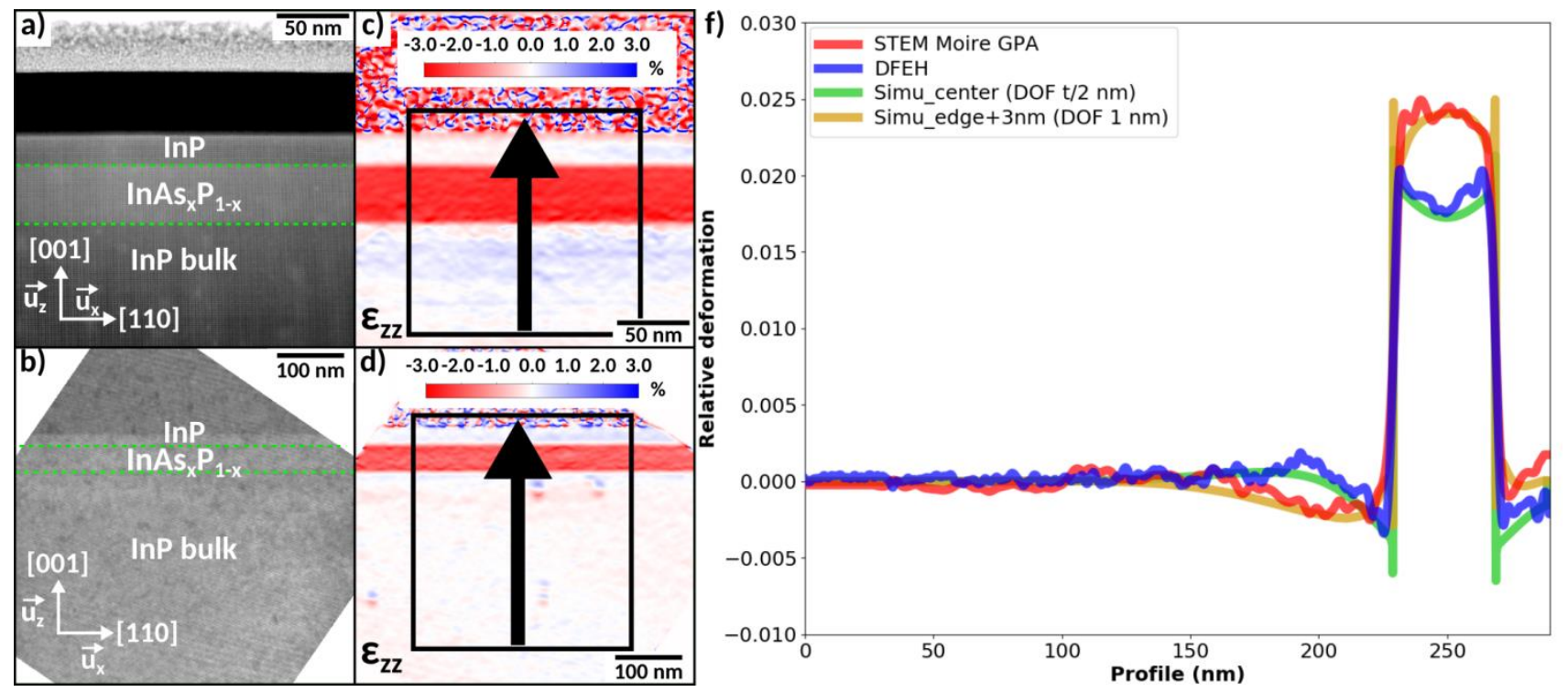

Figure 1. STEM Moiré GPA and DFEH experimental results obtained on a InP/InAs0.35P0.65/InP stack. a) One STEM Moiré hologram with $2048 \times 2048$ pixels and a pixel spacing of $116 \mathrm{pm}$. b) One dark-field electron hologram from the (002) diffracted beam. c) Ezz STEM Moiré GPA deformation map from a). d) EZz DFEH deformation map from b) and another hologram from the (220) diffracted beam (not represented here). f) Ezz deformation profiles along the [001] direction from multiple ezz SMG strain maps (averaged) and multiple Ezz DFEH strain maps (averaged) in red and blue, respectively. The line profiles in yellow and green are obtained from simulations presented in Fig. 2 and show the ezz deformation at $3 \mathrm{~nm}$ of the surface of the lamella and averaged over $1 \mathrm{~nm}$ width and the $\varepsilon z \mathrm{z}$ deformation at the center of lamella averaged over half of the thickness, respectively.
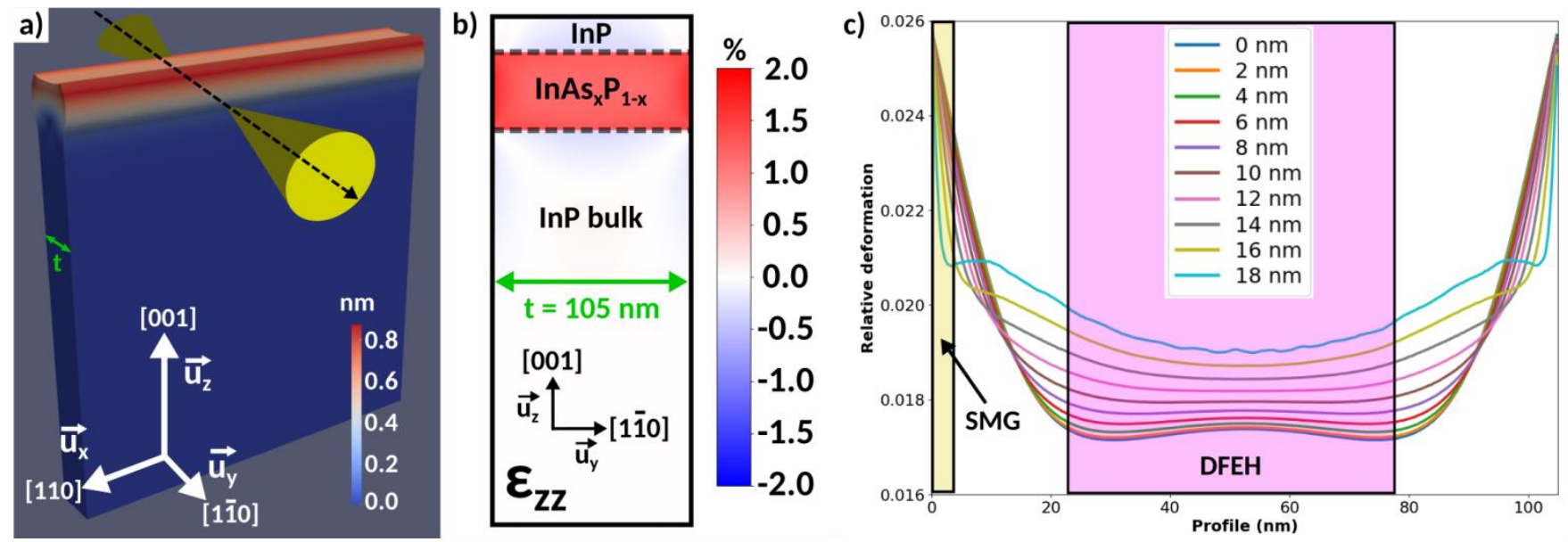

Figure 2. Finite Element Method simulations modelling the strain relaxation from a $105 \mathrm{~nm}$ thick foil. a) Displacement magnitude on the thin foil geometry used for the simulation. The direction of the convergent electron beam is highlighted in yellow and t represents the thickness of the lamella. b) Calculated Ezz relative deformation map from a section of a) perpendicular to the [110] direction using the InP bulk as the unstrained reference. c) $\varepsilon z z$ deformation profiles along the [1-10] direction in the InAs0.35P0.65 layer at different distances from the mid-height position. The approximative depth-of-field and depth location of both STEM Moiré GPA (SMG) and DFEH are highlighted in yellow and purple, respectively.

References 
[1] B.W. Bell, C.L. Koliopoulos, Moiré topography, sampling theory, and charged-coupled devices, Opt. Lett. 9 (1984) 171.

[2] Y. Morimoto, T. Hayashi, N. Yamaguchi, Strain Measurement by Scanning-moire Method, Bull. JSME. 27 (1984) 2347-2352.

[3] D. Su, Y. Zhu, Scanning moiré fringe imaging by scanning transmission electron microscopy, Ultramicroscopy. 110 (2010) 229-233.

[4] S. Kim et al., Scanning moiré fringe imaging for quantitative strain mapping in semiconductor devices, Appl. Phys. Lett. 102 (2013) 161604.

[5] A. Ishizuka, M. Hytch, K. Ishizuka, STEM moiré analysis for 2D strain measurements, J. Electron Microsc. (Tokyo). 66 (2017) 217-221.

[6] A. Pofelski et al., 2D strain mapping using scanning transmission electron microscopy Moiré interferometry and geometrical phase analysis, Ultramicroscopy. 187 (2018) 1-12.

[7] A. Pofelski, S. Ghanad-Tavakoli, D.A. Thompson, G.A. Botton, Sampling optimization of Moiré geometrical phase analysis for strain characterization in scanning transmission electron microscopy, Ultramicroscopy. 209 (2020) 112858.

[8] V. Prabhakara et al., Strain measurement in semiconductor FinFET devices using a novel moiré demodulation technique, Semicond. Sci. Technol. 35 (2020) 034002.

[9] A.Y. Borisevich, A.R. Lupini, S.J. Pennycook, Depth sectioning with the aberration-corrected scanning transmission electron microscope, Proc. Natl. Acad. Sci. 103 (2006) 3044-3048.

[10] E. Javon et al., Dynamical effects in strain measurements by dark-field electron holography, Ultramicroscopy. 147 (2014) 70-85.

[11] A. Pofelski, V. Whabi, S. Ghanad-Tavakoli, G. Botton, Assessment of the strain depth sensitivity of Moiré sampling Scanning Transmission Electron Microscopy Geometrical Phase Analysis through a comparison with Dark-Field Electron Holography, Ultramicroscopy. 223 (2021) 113225.

[12] The authors are grateful to the Natural Sciences and Engineering Research Council for a Discovery Grant supporting this work. 